\title{
Simulation numérique de l’impact d'une souille de matériaux marins sur la stabilité du littoral, en suivant les mesures en bassin de Migniot et Viguier (1979)

\author{
Béryl du Gardin \\ Ifremer Centre de Brest - BP70 - 29280 PLOUZANE cedex - 0282224625 - dugardin@ifremer.fr
}

\section{Résumé:}

Les formules de transport sédimentaire total permettent de reproduire l'évolution observée en cuve, en houle variable, d'une pente de $4 \%$, si on ajoute au transport classique de Bijker, un terme dû à la pente, l'asymétrie et le courant de retour de houle. L'estran reste cependant mal décrit. Pour un fond avec souille, de topographie plus accidentée, une réduction temporelle (durées x3/4) est nécessaire pour reproduire l'évolution de la souille et ses abords.

\section{Abstract:}

Total sedimentary formulae enable to simulate numerically observed topographic evolution of a gentle slope (4\%) in a wave tank, if we add to the classical Bijker formula a transport due to slope, wave asymmetry and wave return flow. (The upper part of the profile is still badly simulated). For an initial slope with a sandpit, where depths vary more quickly, we had to reduce experiment duration $(\mathrm{x} 3 / 4)$ in order to simulate near-field evolution of the pit.

\section{Introduction :}

Migniot et Viguier en 1979 et $1983^{3}$ sont les seuls à avoir quantifié l'impact d'une extraction de granulats marins sur la stabilité du littoral. Les données disponibles en taille réelle ou en bassin (ainsi celles du projet européen SANDPIT) concernent une souille et ses abords, sans instrumentation ou représentation expérimentale du littoral adjacent. Ainsi, malgré les distorsions d'échelles, l'accès difficile aux données (qu'il a fallu numériser) et la complexité des houles, nous simulons ces expériences pour les généraliser ensuite à l'échelle réelle et à des régimes hydrodynamiques différents.

\section{$\underline{\text { 2.Numérisation des données }} \underline{1}$ acquises dans la cuve à houle du LCHF.}

\subsection{Conditions initiales :}

Le profil de plage initial est un plan incliné vers l'ouest de 4\% par rapport à l'horizontale. Le sédiment, de la bakélite, occupe un rectangle entre les altitudes +0.067 à -0.413 à m par rapport au niveau moyen de la surface de l'eau. Une souille de même géométrie est creusée successivement à $12 ; 18 ; 24,5$ et $31 \mathrm{~cm}$ sous ce niveau moyen (voir figure 1).

\subsection{Marée}

Une marée sinusoïdale est effectuée par remplissage du bassin depuis l'ouest. Sa période est 15 minutes et son marnage $0,054 \mathrm{~m}$. 


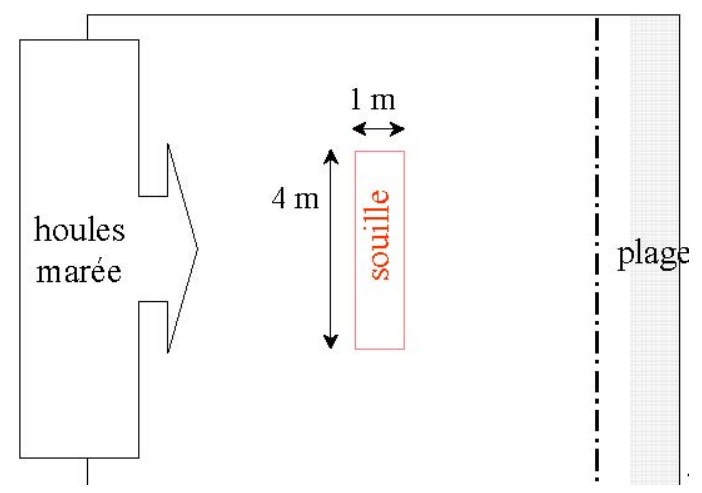

Figure 1 : Cuve à houle, exemple de la souille à $-18 \mathrm{~cm}$. Bakélite : densité 1,38 ; granulométrie $D 50=375 \mu \mathrm{m}$; $D 10=0,67 D 50 ; D 90=1,4 D 50$; occupe un rectangle de $12 \mathrm{~m} \mathrm{~W}$-E, 9,5 $\mathrm{m} \mathrm{N}$-S. Souille : $8 \mathrm{~cm}$ de surcreusement,

flancs à $26 \%$, occupation totale

$1 \mathrm{~m}$ x 4m, parallèle à la côte. 01 BM HM13

\subsection{Houles: trains de dix houles}

Une houle sinusoïdale est initiée depuis l'ouest. Elle est formée par des trains de 10 vagues. Seul un enregistrement de $4 \times 130$ vagues individuelles initiées par ce batteur à houle a été conservé (Migniot et al. ${ }^{4}$ et figure 2). D'après ces données, si $H_{1 / 10}$ est la hauteur quadratique moyenne des $10 \%$ de plus hautes vagues, alors la hauteur quadratique moyenne globale est $H_{\mathrm{rms}}=0,60 H_{1 / 10}$ Lesvagues extrêmes sont Hmin=0,25 H1/10 et Hmax=1,20 H1/10.

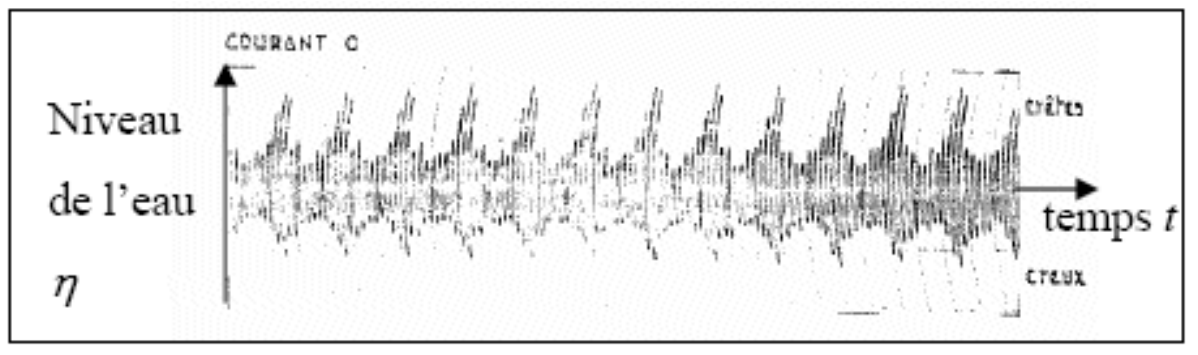

Figure 2 :exemple d'enregistrement de 130 vagues individuelles. 4

\subsection{Houles : dispersion angulaire}

Le batteur à houle oscille de $5^{\circ}$ de part et d'autre de sa direction moyenne. Nous l'avons quantifié à partir des mesures en aval d'un brise-lames dans cette cuve à houle (Manougian et Lalaut $)^{5}$. Ainsi, ces oscillations se traduisent, dans le modèle de houle spectral SWAN utilisé (décrit plus loin), pour les zones non directement abritées, par une dispersion angulaire de $8^{\circ}$ (erreur relative : $2 \%$ ).

Remarquons que dans les zones immergées abritées, SWAN sous-estime la houle car il ne modélise pas la diffusion de l'onde de houle aux extrémités de l'obstacle. Ceci n'est pas pénalisant ici, où de telles zones ne sont pas présentes. 


\subsection{Houles : cycle de 5 heures}

Différentes houles ont été appliqués successivement, lors d'un cycle de 5 heures (tableau cidessous). Ainsi, pour une durée donnée (15 ou $75 \mathrm{~min}$ ), la houle a une période donnée $T$, tandis que sa hauteur $H_{1 / 10}$ définie précédemment ( $§ 1.2$ ) varie entre $H_{1 / 10 \min }$ et $H_{1 / 10 \max }$.

Nous avons calculé pour ces différents intervalles la hauteur de houle significative $H_{\mathrm{s}}(1)$ en supposant une variation sinusoïdale de $H_{1 / 10}$. Nous avons utilisé la relation usuelle : $H_{\mathrm{s}}=2^{0,5} H_{\mathrm{rms}}$.

Nous avons aussi calculé la hauteur significative de houle, sur des intervalles successifs, après avoir réordonné les vagues individuelles par hauteur croissante $\left(H_{\mathrm{s}}(2)\right)$. Cela reflète mieux le contraste entre les différentes vagues lors du calcul de transport sédimentaire, d'autant que nous avons détaillé la description des plus grandes vagues en les regroupant par intervalles de 5 minutes seulement (tableau ci-dessus). En effet, le transport sédimentaire calculé par le logiciel utilisé tient compte uniquement de la hauteur significative de la houle, quel que soit l'étalement du spectre de houle.

\begin{tabular}{|l|l|l|l|l||l|l|l|}
\hline \multicolumn{4}{|l|}{ Houles de l'expérience } & \multicolumn{3}{|l|}{ Houles réordonnées croissantes } \\
\hline $\begin{array}{l}\text { durée } \\
(/ \mathrm{min})\end{array}$ & $\begin{array}{l}\text { Période } T \\
(/ \mathrm{s})\end{array}$ & $\begin{array}{l}H 1 / 10 \mathrm{~min} \\
(/ \mathrm{m})\end{array}$ & $\begin{array}{l}H 1 / 10 \mathrm{max} \\
(/ \mathrm{m})\end{array}$ & $\begin{array}{l}\text { Hs }(1) \\
(/ \mathrm{m})\end{array}$ & $\begin{array}{l}\text { durée } \\
(/ \mathrm{min})\end{array}$ & $\begin{array}{l}\text { Période } T \\
(/ \mathrm{s})\end{array}$ & $\begin{array}{l}H s(2) \\
(/ \mathrm{m})\end{array}$ \\
\hline 75 & 0.92 & 0.013 & 0.040 & 0.0238 & 75 & 0.92 & 0.0107 \\
\hline 75 & 1.15 & 0.013 & 0.040 & 0.0238 & 75 & 1.15 & 0.0176 \\
\hline 75 & 1.39 & 0.013 & 0.027 & 0.0173 & 75 & 1.39 & 0.0281 \\
\hline 15 & 1.15 & 0.040 & 0.067 & 0.0457 & 30 & 1.27 & 0.0404 \\
\hline 15 & 1.39 & 0.040 & 0.067 & 0.0457 & 30 & 1.505 & 0.0559 \\
\hline 15 & 1.62 & 0.040 & 0.067 & 0.0457 & 5 & 1.62 & 0.0737 \\
\hline 15 & 1.39 & 0.067 & 0.093 & 0.0679 & 5 & 1.62 & 0.0854 \\
\hline 15 & 1.62 & 0.067 & 0.093 & 0.0679 & 5 & 1.62 & 0.1121 \\
\hline
\end{tabular}

\subsection{Conditions représentées à échelle réelle}

Les expériences décrites ici visaient à reproduire les conditions naturelles du littoral du Golfe de Gascogne. Cette représentativité reste à démontrer, à cause des distorsions d'échelle inhérentes à tout modèle réduit (discutées par du Gardin et al. ') et à cause du régime de houle appliqué, moins contrasté en hauteurs comme en périodes que les houles naturelles (tableau cidessous).

\begin{tabular}{|l|l|l|}
\hline Caractéristiques des houles & expérience & littoral réel 7 \\
\hline \hline Hauteur maxi $(2 \%$ annuel $) / H_{\text {rms }}$ & 5 & 8 \\
\hline Période maxi $(2 \%$ annuel $) /$ période mini $(2 \%$ annuel $)$ & 2 & 4 \\
\hline
\end{tabular}


Session 2: Dynamique sédimentaire et transports de particules

Voici pour illustration les conditions naturelles reproduites :

\begin{tabular}{|c|c|c|}
\hline grandeur & échelle & Exemple en taille « nature » ou explication. \\
\hline Dimensions horizontales & 200 & \\
\hline Dimensions verticales & 75 & $\begin{array}{l}\text { Marnage } 3,7 \mathrm{~m} \text { (qui serait coef. } 80 \text { ). Souilles } \\
6 ; 11 ; 16 ; 21 \mathrm{~m} \text { sous BM coef. } 100 .\end{array}$ \\
\hline $\begin{array}{l}\text { Vitesses de houle } \\
\text { (vitesse orbitale et } \\
\text { courant de retour) }\end{array}$ & 8,66 & $\begin{array}{l}\text { conforme à la similitude de Froude. Les } \\
\text { hauteurs et longueurs de houle sont à } \\
\text { l'échelle } 75 \text {. }\end{array}$ \\
\hline Courant de marée & 4 & Non-conforme (Froude) \\
\hline Temps sédimentaires & $\begin{array}{lr}25 & \mathrm{X} \\
70 & (2) \\
(3) & \\
\end{array}$ & $\begin{array}{l}1 \text { cycle ( } 5 \mathrm{~h} \text { en cuve) mime un an « nature » } \\
\text { sur sable quartzeux fin }\left(D_{50}=250 \mu \mathrm{m}\right) .\end{array}$ \\
\hline
\end{tabular}

(1)

(2) Le sédiment, pour rester non-cohésif, ne peut subir de réduction d'échelle. Un coefficient empirique de mobilité (x25) de la bakélite a été établi par Migniot et Viguier par comparaison entre expérience et observations du transit (1) sédimentaire longitudinal du littoral des Landes.

Migniot et Viguier justifient ce courant de marée par un coefficient de mobilité de la bakélite qui serait différent pour les houles et pour les courants.

\section{Equations utilisées par le modèle numérique}

\subsection{Marée}

La marée génère des courants, faibles ici, dont la moyenne verticale est calculée selon les équations de Saint-Venan, suivant un schéma ADI, par le module FLOW du logiciel Delft3D. A l'Ouest, est appliqué un courant sinusoïdal d'amplitude $0,0047 \mathrm{~m} . \mathrm{s}^{-1}$, de période $15 \mathrm{~min}$. Aux autres frontières, le flux est nul.

La viscosité dynamique horizontale $v_{\text {calc }}$ récapitule la viscosité dynamique réelle $v_{d y n}$, la viscosité turbulente de houle $v_{\text {turb }}$ et compense la viscosité numérique $v_{\text {num }}: v_{c_{\text {calc }}-1} v={ }_{\text {dyn }}+v_{\text {turb }}$ $-v_{\text {num }}\left(c f\right.$. Annexe). Cette dernière $\left(v\right.$ num) est prépondérante. $v_{\text {calc }}$ varie de $0,3 \mathrm{~m} . \mathrm{s}$ sur l'estran à $5,5 \mathrm{~m} . \mathrm{s}$ au large.

\subsection{Houles : conservation de l'action}

La houle du large est propagée selon l'équation de conservation de l'action tout en étant modifiée par réfraction sur les fonds et les courants (Hasselmann ${ }^{8}$ ). Un plan de houle stationnaire est ainsi calculé par haute mer, basse mer, maxima de flot et de jusant. Ces plans de houle sont interpolés aux temps intermédiaires.

La dissipation des houles par frottement sur le fond a été calculée selon la formulation de Madsen ${ }^{9}$ (longueur de rugosité 0,25 mm).

Le déferlement des vagues a été modélisé par plafonnement du rapport hauteur de vague $(H) /$ hauteur d'eau $(h)$ (Battjes et Janssen ${ }^{10}$ ). Ce plafond vaut 0,48 quand les vagues du large sont 
inférieures à $0,03 \mathrm{~m} ; 0,76$ quand elles sont supérieures à $0,067 \mathrm{~m} ; 0,53$ dans le cas intermédiaire (Migniot et al. ${ }^{4}$.

\subsection{Transport sédimentaire classique: formule de Bijker ${ }^{2}$}

Cette formule donne le transport sédimentaire sous l'effet combiné des houles supposées symétriques et du courant de marée. Elle est implémentée dans le module TRAN du logiciel Delft3d; le coefficient multiplicatif $b$ y varie de 5 en eau peu profonde à 2 en eau profonde, avec une transition linéaire en fonction de $H_{r m s} / h$, qui a lieu quand ce rapport vaut entre 0,4 et 0,05 .

\subsection{Transport sédimentaire additionnel :}

Un transport additionnel, surtout transversal, a été évalué selon Bailard " dans le module TRAN du logiciel Delft3d, rendant compte d'effets de pente, d'asymétrie des vagues, voire de courants à renseigner. L'asymétrie des vagues y est évaluée selon la hauteur d'eau et la houle locales (Roelvink et Stive ${ }^{12}$ ).

Le courant de retour de houle uret y a été renseigné, évalué au premier ordre à partir de la théorie linéaire de houle selon Stive et Battjes ${ }^{13}$. Le flux massique d'eau $M$ à la vitesse de ce courant de retour égale la densité d'énergie de houle $E$ divisée par la célérité $c$ des ondes de houle ( $\mu$ est la masse volumique de 1'eau) :

$$
M=\mu . h . u_{\mathrm{ret}}=E / c \text {, soit } u_{\mathrm{ret}}=\left(g \mathrm{Hrms}^{2}\right) /(8 c h) \text {. }
$$

\section{Résultats (à l'échelle de la cuve à houle):}

\subsection{Résultats expérimentaux sans souille, de Migniot et Viguier}

En l'absence de souille, le régime hydrodynamique appliqué conduit à une érosion du haut du profil et une sédimentation en eau plus profonde. La zone d'érosion s'étend du haut d'estran $(z=0,027 \mathrm{~m})$ jusqu'à la profondeur $z=-0,1 \mathrm{~m}$, avec deux maxima locaux d'érosion, en bas d'estran $(z=-0,027 \mathrm{~m})$ et plus profondément, en $z=-0.083 \mathrm{~m}$. La zone de sédimentation a un maximum local
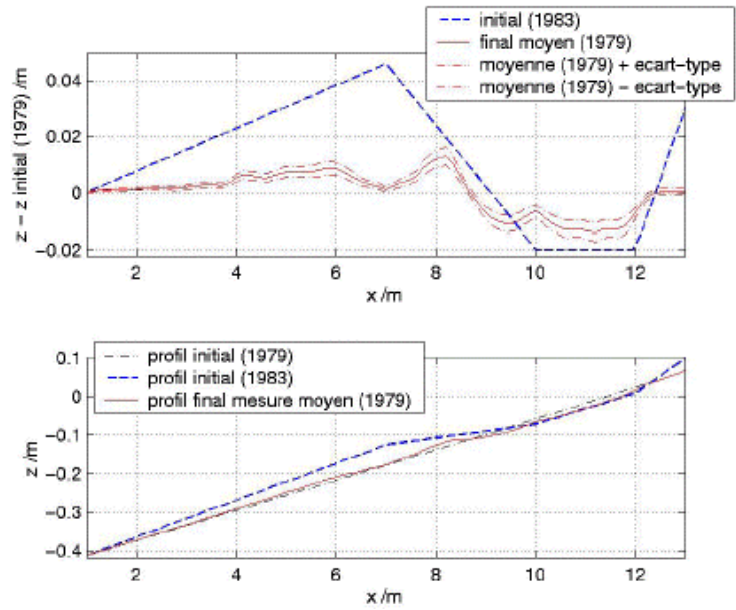

Figure 3 : Evolutiondes fonds expérimentaux,initialement sans souille, après deux cycles de houle. Les mesures étaient exprimées à échelle nature (X, Z), où en basse mer de vive-eau (coef. 100), $Z=0$ et $X=X B E=500$ $m$. Elles sont traduites à l'échelle de l'expérience $(x, z)$, où $z=0$ est le niveau moyen de l'eau, soit : $x=10,5+(X B E-$ $X) / 200$ et $z=-0.033+Z / 75$. 
Ce résultat est nouveau. Les cinq profils d'une cinquantaine de points chacun, relevés après quatre expériences identiques de deux « cycles annuels » sur un fond sans souille, n'avaient jamais été numérisés et moyennés. Ce résultat est significatif car l'écart-type vaut moins de la moitié de la variation moyenne de la topographie. Par ailleurs, le profil initial choisi en 1983 (Migniot et al. ) est dit similaire au profil final sans souille de 1979 , alors qu'il surestime largement les volumes déplacés (érosion x1,5 ; sédimentation x6).

Le profil initial sans souille (1979) n'a pas été mesuré. Celui de la figure 4 est le profil rectiligne prévu, que nous avons incurvé légèrement vers le bas pour

0,5

obtenir un bilan sédimentaire nul. Cette incurvation vaut $\mathrm{d} z=-4.10^{-4}(x-1)(13-x)$, d'amplitude maximale $6,6 \mathrm{~mm}$, à mi-profil. Les simulations numériques utilisent le profil initial prévu, rectiligne. Leur résultat est comparé, dans la suite, aux profils expérimentaux redressés, c'est-àdire corrigés de leur incurvation.

Pour les expériences avec souille, la mesure du profil initial réel, faite au voisinage de la souille, confirme cette incurvation, car elle montre des fonds légèrement plus bas que prévu à une abscisse donnée.

\subsection{Calibrage du transport additionnel (cas sans souille)}

Nous avons adopté les coefficients suivants, 0,11 pour l'effet de pente et 0,4 pour l'asymétrie des vagues, conformément à Nipius ${ }^{14}$. Le facteur multiplicatif sur l'effet du courant de retour a été fixé à 0,8 pour avoir une bonne localisation de la sédimentation. S'il vaut entre 0,5 et 0,7 , il apparaît une zone fortement ridée vers $x=8 \mathrm{~m}$. S'il vaut plus de 0,9 ou moins de 0,5 , la sédimentation est déplacée respectivement vers le large ou la côte.
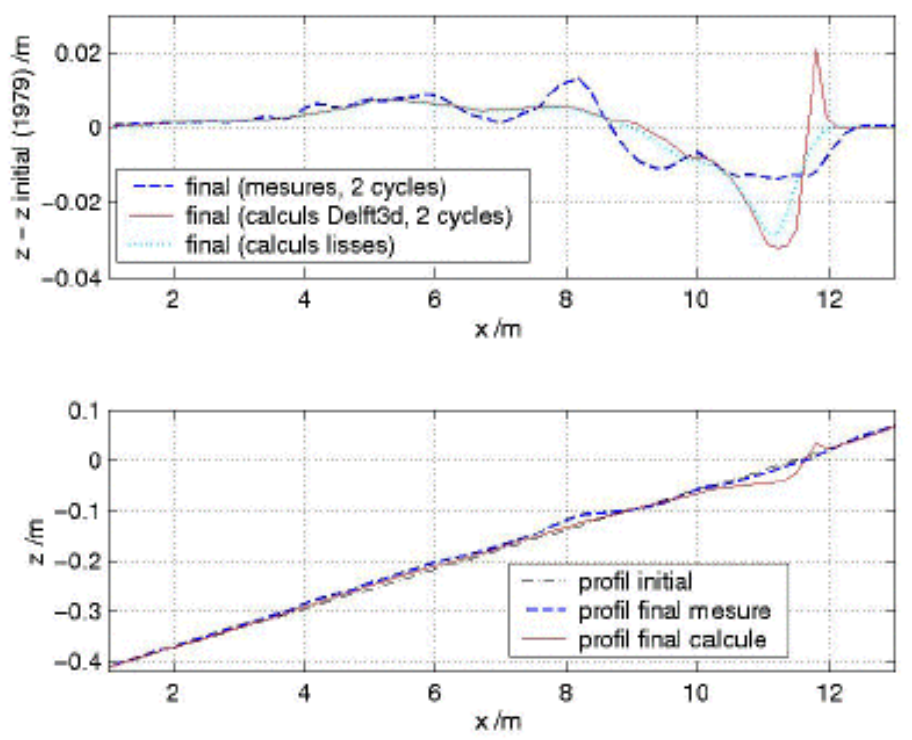

Figure 5 : Comparaison entre profil mesuré, ou calculé avec Delft3d (voir texte), dans le cas d'un profil sans souille.

La topographie de l'estran est très mal prédite ; un lissage de la terrasse supérieure a été ajouté en fin de chaque cycle. Ce lissage oblige une pente d'estran finale supérieure ou égale à la pente initiale (4\%). 
Pour conformer le volume déplacé aux observations (figure 5), les transports sont multipliés (x20), via une dilatation de la durée de chaque houle. La topographie calculée en zone profonde concorde très bien. En profondeur intermédiaire $(x=8 \mathrm{~m})$, le volume sédimenté calculé est correct mais plus étalé.

Remarquons que la dilatation temporelle appliquée est proche du facteur empirique (x25) de Migniot et Viguier. En effet, les formules de transport employées utilisent la vitesse de chute et la densité du sédiment (soit ici la bakélite, chutant à $0,02 \mathrm{~m} . \mathrm{s}^{-1}$ ), mais elles ont été établies pour du quartz et nécessitent des corrections avant d'être appliquées à d'autres sédiments.

Il s'agit bien ici du calibrage du transport additionnel. En effet ce transport est prépondérant devant celui de Bijker seul, sur fond sans souille.

\subsection{Sensibilité de ce transport additionnel à l'ordre des vagues}

Les résultats présentés au $\S 3.2$ utilisent le cycle de houle $H_{\mathrm{s}}(2)$ (voir $\S 1.4$ ) où les plus fortes vagues sont décrites explicitement. Avec le cycle de houle $H_{\mathrm{s}}(1)$, une sédimentation suffisante par faible profondeur (en $z=-0.13 \mathrm{~m}$ ) est obtenue avec la même dilatation temporelle (x20), mais la sédimentation en eau plus profonde est alors trop ténue (moins de $0,002 \mathrm{~m}$ sédimentés sous $Z^{=-}$ $0.25 \mathrm{~m}$ ). Ainsi, le transport sédimentaire n'est pas déterminé par l'énergie moyenne de la houle, mais par la hauteur des vagues individuelles, qu'on peut réordonner artificiellement pour conserver un temps de calcul raisonnable.

\subsection{Cas avec souille}

Le modèle de transport total, calibré ci-dessus, surestime le remplissage d'une souille à -18 $\mathrm{cm}$. Nous l'expliquons par un relief trop rapidement variable devant les conditions hydrodynamiques. Le temps de chute d'une particule de bakélite au fond de la souille (13 s) est en effet non négligeable devant le temps de traversée de cette souille (31 s) par les courants de retour de houle $\left(0,032 \mathrm{~m} . \mathrm{s}^{-1}\right.$ pour les plus fortes houles). Une diminution de la dilatation temporelle (x3/4) permet d'obtenir une évolution de la souille conforme aux observations, avec cependant un décalage de la souille d'environ $15 \mathrm{~cm}$ vers le large.

La sédimentation au large de la souille serait, selon les mesures, (figures 5 et 6), plus importante avec souille que sans elle. Cela n'a aucune explication physique. Il s'agit probablement d'une irrégularité du fond présente dès le début d'expérience. Au large de la souille, les mesures sont donc trop bruitées pour pouvoir être valablement comparées au résultat du modèle numérique. 

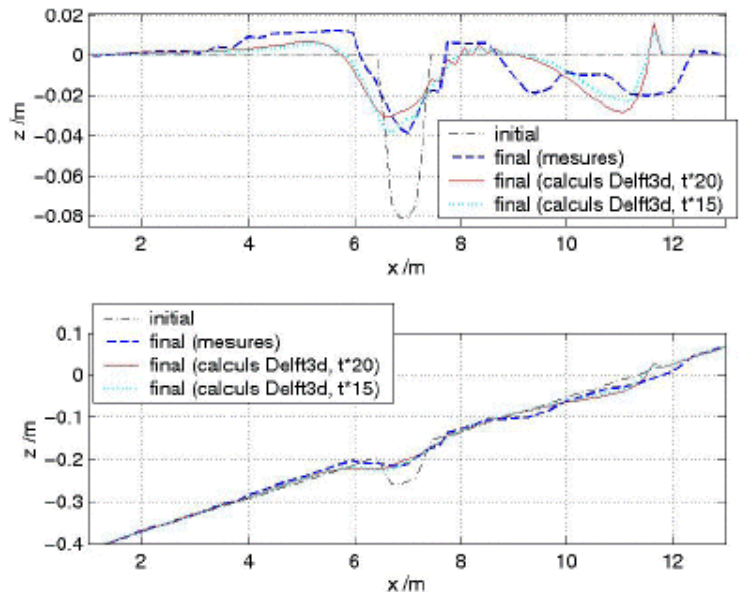

Figure 6 : Evolution au bout de 2 cycles de 5 h du profil avec souille à $-18 \mathrm{~cm}$.

Comparaison entre mesures et calculs.

Le haut de profil ( $\mathrm{z}>-0,1 \mathrm{~m})$ est mal prédit (cf figure 5).

Les mesures, sur 3 profils globaux et 5 profils locaux, passant tous par la souille, sont moyennées ici ; l'écart-type vaut la variation topographique moyenne, sans excéder $2 \mathrm{~cm}$.

\section{Conclusion :}

Nous avons modélisé les transports sédimentaires transversaux en présence d'une houle variable, en utilisant une formulation par transport total. Ce modèle est validé et calibré, pour le domaine sub-tidal, à partir d'expériences en bassin sur une pente uniforme. Il s'applique aussi en présence d'une souille, moyennant une réduction temporelle (x3/4).

\section{Remerciements :}

Merci à J.A. Roelvink (Delft Hydraulics) qui a introduit, pour ce travail, le courant de retour de houle dans le logiciel Delft3d, ainsi que pour ses conseils. Merci à C. Augris et F. Cayocca, de l'Ifremer, qui ont orienté ce travail et à $\mathrm{A}$. Porchon, du CETMEF, qui m'a procuré des documents du LCHF.

\section{Annexe: calculs de viscosité}

La viscosité turbulente a été calculée par : $v_{\text {turb }}=l u_{*}$ où $l=d x=\mathrm{m} 150$ est la plus petite discrétisation spatiale (selon, $\mathrm{x}$ ) et où $\mathrm{u}^{*}$ est la vitesse turbulente au fond due au mouvement orbital de houle. $\mathrm{u}^{*}$ est calculé selon Soulsby et al. ${ }^{15}$ en supposant la houle linéaire et la longueur de rugosité $z 0=0,25 \mathrm{~mm}$. La viscosité turbulente vaut $0,005 \mathrm{~m}^{2} . \mathrm{s}^{-1}$ sous $3 \mathrm{~mm}$ d'eau. Elle vaut 0,018 à $0,021 \mathrm{~m}^{2} . \mathrm{s}^{-1}$, selon la hauteur des houles, au large.

La viscosité numérique a été calculée par :

$v_{\text {num }}=\sqrt{g h} \mathrm{~d} x(1-\mathrm{d} t \sqrt{g h} g h \mathrm{~d} x(1-\mathrm{d} t g h / \mathrm{d} x) / 2$ où $\mathrm{d} x=0,15 \mathrm{~m}$ est la discrétisation spatiale dans la direction des courants de marée et où $\mathrm{d} t=3 \mathrm{~s}$ est le pas de temps hydrodynamique, pour les calculs en schéma ADI. Notons que $\sqrt{g h}$ gh approxime la célérité de la propagation horizontale des ondes de gravité en eau peu profonde. ( $\mathrm{d} t \sqrt{g h} g h$ )/d $x$ approxime donc le nombre de Courant $C$. La viscosité numérique varie de $-0,3$ ( $3 \mathrm{~mm}$ d'eau) à $-5,5 \mathrm{~m}^{2} . \mathrm{s}^{-1}$ (au large). Elle est négative ; autrement $\operatorname{dit} C>1$ (Il atteint 40). 
Ainsi le calcul, pendant le temps $\mathrm{d} t$, transmet l'information à la maille suivante, éloignée de $\mathrm{d} x$, plus vite que la propagation réelle de cet ébranlement. Le schéma ADI et la compensation de la viscosité numérique permettent en effet de mener des calculs stables avec $C>1$.

\section{Références}

1.Migniot C., J. Viguier, 1979. Etude de l'influence de dragages sur l'équilibre des fonds littoraux. Rapport LCHF pour CNEXO, CETMEF, SCTPVN, 58p+figs.

2.Bijker, E.W., 1992, Mechanics of sediment transport by the combination of waves and current, in : Design \& reliability of Coastal Structures, Venice, 1992, 147-173.

3.Migniot C., J. Viguier, J.M. Roques, 1983. Analyse par simulation des effets sur le littoral de l'ouverture de souilles destinées à l'extraction de granulats marins. Rapport LCHF pour CETMEF, SCTPVN, CNEXO, SNAEMM, 46p+figs.

4.Migniot C., J. Viguier, J.M. Roques, 1984. Action simultanée de la houle et des courants sur les mouvements sédimentaires. Rapport LCHF pour Min. mer, SCTPVN, CNEXO. 67p+figs.

5.Manougian, S., Y. Lalaut, 1987. Ouvrages de protection d'un littoral sableux. Rapport LCHF pour CETMEF, SCTPVN. 65p+figs.

6.du Gardin, B., R. Armède, F. Cayocca, C. Augris, 2002. Effets de l'extraction des matériaux marins sur la stabilité du littoral. Ressources Minérales et océan profond, Les Techniques de l'Industrie Minérale 15, Soc. Ind. Min., Paris.

7.CETMEF : données de houle CANDHIS, www.cetmef.equipement.gouv.fr. site de Bayonne, 1989 à 1994.

8.Hasselmann, K., 1974. On the spectral dissipation of ocean waves due to whitecapping. Bound.-layer Meteor., 6 (1-2), 107-127.

9.Madsen, O.S., Y.K. Poon, H.C.Graber, 1988. Spectral wave attenuation by bottom friction: Theory. Proc. 21 th Conf. Coastal Eng., ASCE, 492-504.

10.Battjes, J.A., J.P.F.M. Janssen, 1978. Energy loss and set-up due to breaking of random waves. Proc. of the $16^{\text {th }}$ Int. Conf. Coastal Eng., ASCE, 569-587.

11.J.A. Bailard (1981). An Energetic Total Load Sediment Transport Model for a Plane Sloping Beach. Journal of Geophys. Res., 86 (C11), 10938-10954.

12.Relvink, J.A., and Stive, M.J.F. (1989). Bar-Generating Cross-Shore Flow Mechanics on a Beach. J. Geophysical Research, 94 (C4), 4785-4800.

13.Stive, M.J.F. and Battjes, J.A. (1984). A model for offshore sediment transport. Proc. of the $19^{\text {th }}$ Int. Conf. on Coast. Eng., New York, ASCE, 19221934.

14.Nipius, K.G.(1998). Dwartransportmodellering m.b.v. Bailard toegepast op de Voordelta Grevelingen-monding. Msc Thesis, Delft University of Technology.

15.Soulsby, R.L., Hamm, L., Klopman, G., Myrhaug, D., Simons, R.R., Thomas, G.P., 1993, Wave-current interaction within and outside the bottom boundary layer, Coastal Engineering, 21(1-3), 41-69. 